\title{
Does lipopolysaccharide-based neuroinflammation induce microglia polarization?
}

\author{
Daniel Hernandez-Baltazar ${ }^{1}$, Rasajna Nadella ${ }^{2}$, Abril Alondra Barrientos Bonilla ${ }^{3}$, Yazmin Montserrat Flores Martínez ${ }^{4}$, \\ Abraham Puga Olguín ${ }^{5}$, Paola Heman-Bozadas ${ }^{6}$, María de Jesús Rovirosa Hernández ${ }^{6}$, Iliana Tamara Cibrián Llanderal ${ }^{6}$ \\ ${ }^{1}$ CONACyT-Instituto de Neuroetología, Universidad Veracruzana, Xalapa, Veracruz, Mexico, ${ }^{2}$ IIIT Srikakulam, Rajiv Gandhi University \\ of Knowledge Technologies (RGUKT), India, ${ }^{3}$ Centro de Investigaciones Biomédicas, Universidad Veracruzana, Xalapa, Veracruz, \\ Mexico, ${ }^{4}$ Programa Institucional de Biomedicina Molecular, Escuela Nacional de Medicina y Homeopatía, Instituto Politécnico \\ Nacional, Ciudad de México, Mexico, ${ }^{5}$ Instituto Interdisciplinario de Investigaciones de la Universidad de Xalapa, Xalapa, Veracruz,

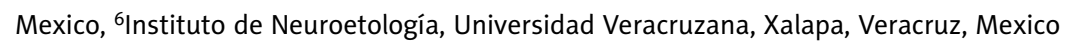

\begin{abstract}
Lipopolysaccharide (LPS) is a potent immunogen when administered locally and/or systemically. The peripheral immunization with LPS could contribute to the progression of neurological diseases because a strong link between neuroinflammation and dopaminergic degeneration has been found. The switch between the survival and neuronal death in substantia nigra could be related to $M 1$ (neurotoxic) and $M 2$ (neuroprotective) microglia phenotypes. In this review, we present the current findings about microglia roles, biomarkers, and natural or synthetic immune modulators determined in the LPS-based murine model.
\end{abstract}

Key words: cell signalling, lipopolysaccharide, proliferation, microglia cells, neuroinflammation.

\section{Introduction}

Neuroinflammation is defined as an inflammatory response mediated by the production of cytokines (tumour necrosis factor $\alpha$ (TNF- $\alpha$ ), interleukin (IL)-1 $\beta$, IL-4, IL-6), chemokines, mitochondrial nicotinamide adenine dinucleotide phosphate (NADPH) oxidase, inducible nitric oxide synthase (iNOS), reactive oxygen species (ROS, superoxide, hydroxyl radicals); and gliosis (astrocytes and microglia) in the central nervous system (CNS) and in the peripheral nervous system (PNS) $[7,24,25,55]$. In this context, the microglia activation has been associated with persistent neuronal damage, changes in long-term potentiation, motor and cognitive dysfunction in neurodegenerative diseases $[9,37,87,96]$. But as per the recent studies, microglia is related to maintenance of homeostasis, modulation of neurogenesis and cognitive processes in the healthy adult brain [3].

To understand the complete cellular and molecular mechanisms underpinning the neuroinflammatory diseases such as Parkinson disease (PD), paraquat, 6-hydroxydopamine (6-OHDA) and lipopolysaccharide (LPS) induced animal models have been used. The paraquat model enables the degeneration of dopaminergic and gamma aminobutyric acid (GABAergic) neurons with a slight presence of inflammation; while the 6-OHDA model, although

\section{Communicating author}

Daniel Hernandez-Baltazar, PhD, CONACyT-Instituto de Neuroetología, Universidad Veracruzana, Xalapa, Veracruz, Mexico,

e-mail: dan.hernandez.baltazar@gmail.com 
it promotes the death of dopaminergic neurons, mainly in the substantia nigra (SN), is accompanied by discrete neuroinflammation at the site of injury $[11,42,74,90]$.

Lipopolysaccharide is an endotoxin found in the cell wall of gram-negative bacteria, which can interact with a variety of cells through the toll-like receptor 4 (TLR4) $[64,69]$. The local or peripheral administration of LPS activates microglia [104] and astrocytes [46], immune cells and macrophages-like cells in different organs of the body $[55,56,83]$. The peripheral injection of LPS-induced endotoxemia favours increased brain levels of interleukins, prostaglandins, nitric oxide, and other modulator substances. Injection of LPS directly into the CNS can induce an inflammatory response similar to peripheral administration $[29,38,81,85]$. Hence, the pathophysiology of PD, neurological disorders related to prenatal systemic inflammation, septic shock, gut-brain axis in maintaining homeostasis [22] and the search for new anti-inflammatory drugs can also be studied using LPS-induced models $[4,5,34,39]$.

\section{Lipopolysaccharide-related murine models}

In the central and peripheral nervous systems, the LPS effects have been evaluated from several doses and two administration routes (local and peripheral). Two to ten $\mu \mathrm{g}$ of LPS has been used for unilateral intracranial injection $[4,8,44,94]$, while doses of 0.05 to $5 \mathrm{mg} / \mathrm{kg}$ have been evaluated in models of intraperitoneal injection $[13,17,87]$, highlighting the LPS derived from Escherichia coli, serotype 055:B5 as the most used one in preclinical research. With regard to main LPS effects, the intracranial injection of this immunogen produces dose-dependent microglial activation and increases neurodegeneration in susceptible brain nuclei (e.g. $\mathrm{SN}$ ) in mice and rats $[4,8,30,44,55,56,93]$. Furthermore, depending on the LPS that is injected in SN [24,78], globus pallidus [102], neostriatum [19] or lateral ventricles [43], the microglial activation is indistinctly characterized by morphological changes, cellular stress and antiand pro-inflammatory cytokine production. On the contrary, the intraperitoneal injection of LPS in adult rats (LPS-induced endotoxemia) causes microglia migration (and priming) in a dose-dependent manner in substantia nigra (pars compacta, compacta dorsal and pars reticulata) and damage in peripheral organs $[10,13,17,87]$; while in mice the chronic
LPS administration induces motor deficits caused by alterations in the dopaminergic neurons of SN $[16,50,65]$, and cellular stress in organs [72]. In addition, LPS promotes loss of dopaminergic neurons of neonatal rats when their mothers receive the intraperitoneal administration of this immunogen on day 9.5 [39] or 10.5 prenatal [110].

\section{Lipopolysaccharide and microglia polarization}

Lipopolysaccharide has been used as a classical toxin to activate immunocompetent cells and trigger a systemic and central inflammatory response $[56,96]$. LPS consists of a lipid portion called lipid A, a lipopolysaccharide $\mathrm{O}$ (core) and an oligosaccharide $\mathrm{O}$. Lipid $A$ is responsible for the activation of macrophages and the production of pro-inflammatory cytokines. The polysaccharide $O$ gives the bacteria its serological specificity, and the oligosaccharide $O$ is responsible for linking the polysaccharide $O$ to the lipid A [84]. The LPS binds to the specific receptor complex CD14/TLR4/LBP that is found in the membrane of microglial cells and induces their activation. The LPS, once recognized by the membrane-associated CD14/TLR4/LBP receptor complex, transports the lipid A to the MD-2 protein, which can be found soluble or bound to TLR4 through hydrogen bonds [1,54]. The formation of this complex is the key for the initiation of intracellular signalling.

Microglia are the resident mononuclear phagocytes in the CNS that belong to the non-neuronal glial cells [36]. Microglia change their morphology on activation from the inactive or resting state due to the CNS damage caused by infections, brain trauma, injury, ischemia, etc. The activated microglia exist either in the classical M1 phenotype or alternative M2 phenotype and these two phenotypes are the two activated states that represent their extreme biological functional states and determine their physiological characteristics. The activated classical M1 phenotype is seen due to the presence of high levels of interferon (IFN), tumour necrosis factor (TNF) and LPS and in turn produces and releases inflammatory mediators that are toxic to the neurons, thus leading to the neuroinflammation or neurodegeneration and damage [26]. On the contrary, the activated alternative $M 2$ phenotype is seen due to the presence of high levels of interleukins (IL-4, IL-13) and in turn produces and releases anti-inflammatory cytokines and 
various neurotrophic factors that protect and regenerate the neurons, thus leading to the neuroprotection and recovery [61].

As per the recent studies, M1 and M2 phenotypes can be distinguished basing on inflammation-related cytokines [48], specific cell membrane proteins and arginine metabolism-associated molecules [97]. LPS is a TLR4 agonist that can polarize the microglia into the M1 phenotype and also effectively induces inflammatory responses, thus reducing the $\mathrm{M} 2$ phenotype and its anti-inflammatory markers [59]. This is the reason behind selecting LPS-induced neuroinflammation in various studies. LPS-induced neuroinflammation is mediated by TLR4/nuclear factor (NF) $-\kappa B$ signalling pathway and the expression of $N F-\kappa B$ is crucial for the M1 phenotype of microglia [103]. In order to cure neuroinflammation, several researchers have proposed different sources but the common solution among all that research includes the inhibition of the $M 1$ phenotype and promotion of the $M 2$ phenotype by suppressing TLR4/NF- $\mathrm{B}$ pathways $[79,101,105]$. Even though several models exist for neuroinflammation, LPS is the best and most widely used one [6], especially as those including the study of microglia as LPS play a key role in microglia polarization.

In vitro and in vivo studies have suggested that LPS exerts microglia polarization as evidence of microglia plasticity $[68,91]$. For example, the unilateral microinjection of $5 \mu \mathrm{g}$ of LPS in SNpc of male rats induces an increase in the $M 1$ phenotype, which was associated with the loss of dopaminergic neurons at 1,3 and 7 days post-injection. This neurotoxic event was evidenced by high levels of oxidative stress, upregulated expression of iNOS, cyclooxygenase 2 (COX-2) and nitrotyrosine, and downregulated expression of arginase 1 . However, facts were reversed with capsaicin, where a neuroprotective effect, characterized by the M2 microglial phenotype, is increased, arginase 1 expression is increased and low levels of oxidative stress markers in rat SN was determined [8]. In the female rats, the single administration of $5 \mu \mathrm{g}$ of LPS into SNpc favours loss of dopaminergic neurons at 7 days after microinjection. In the first $24 \mathrm{~h}$ post injection of LPS, a neurotoxic effect was evidenced by M1 microglial activation, accumulation of fluorescent oxidized hydroethidine, upregulated proinflammatory markers (NF- $\kappa B$ p-p65), inflammatory markers (TNF- $\alpha$, $\mathrm{IL}-1 \beta$ and PEG2) and oxidative stress markers (COX-2 and iNOS). However, this fact was reversed with pre-treatment by immune modulators such as somatostatin, where an increased level of $M 2$ polarization and low levels of neuronal death or oxidative stress was observed [4]. Additionally, the conversion of M1 to $M 2$ polarization is determined using treatments with dexamethasone [12], Ginsenoside Rg1 [88], G1 agonists [62] or polydatin [44].

A lot of evidence has been used to support that LPS favours the M1 microglia phenotype, because of increased CD86, IL-1 $\beta$, CCL5, IL-6, iNOS, and CXCL1 mRNA levels, but it did not induce significant changes in Arg1, CD06, IGF-1 and PPARg mRNA levels, which are microglia phenotype markers [89]. Some studies suggested that such factors as dosage and combination of LPS with other stressors can alter the $M 1 / M 2$ switch $[17,80]$. In this context, a study performed by Rabenstein et al. [73] reported that the microglia polarization is a derivative of LPS, or LPS with a cell pre-conditioned by ischemia and in a primary microglia culture obtained from pup rats; in the $10 \mathrm{ng} / \mathrm{ml}$ LPS group, the polarization to the $M 1$ microglial phenotype was detected, while that in the group with LPS and pre-conditioning level increased of IGF1, an anti-inflammatory factor [40], was found. Serdar et al. [76] injected intraperitoneally $0.1 \mathrm{mg} / \mathrm{kg}$ LPS followed by hypoxic ischemic brain injury in rats; this study demonstrated reduction in neuronal density, microglial activation on hippocampus and cerebral cortex, changes in gene expression profiles associated with M1/M2 microglial polarization. They observed a significant increase in the gene expression levels of iNOS, IL-1 and IL- 6 in the LPS group. However, in the LPS/hypoxic ischemic brain injury group, the increase in these molecules was more significant, showing that even if LPS is a powerful microglial activator, the polarization of these cells is closely related to the simultaneous events that occur in the body.

\section{M1 and M2 microglia, cell signalling and modulators}

The level of activation of microglial cells depends on the intensity, duration and type of the stimulus and can generate a neuroprotective or neurotoxic response [30,31]. At the level of the CNS and PNS, two phenotypes of microglia, $M 1$ and $M 2$, have been distinguished. The M1 phenotype (neurotoxic) is characterized by the release of pro-inflammatory factors from thin branched microglia that can be 
derived to amoeboid (typical hallmark of neurodegeneration); while $M 2$ microglia retains branched morphology and promotes the release of anti-inflammatory factors leading to neuroprotection [8,92] (Fig. 1). These morphological changes have been associated with the expression of the genes $\mathrm{Ccr} 2$, Nox2, TNF, Ly6c, IL12a, IL12b and Icam1 [17]. To assess microglial activation, markers such as ionized calcium binding adapter molecule-1 (Iba-1) [44], cluster of differentiation 68 (CD68) [67], CD11b or CD11b/c [8], CD206 [2] and complement receptor type 3 (CR3) [109], which is commonly named as OX42 [30], have been used. Among these markers, the most common ones are CD16/32 and CD86 for the M1 subtype, and CD206 and CD11b for M2 microglia [108] (Fig. 2).
The $M 1$ and $M 2$ microglia phenotypes exhibit different intracellular signalling. In Figure 2, the interaction of anti- or pro-inflammatory mediators to define microglia roles is illustrated. In the case of M1 microglia, the binding of LPS to the MD2/TLR4 induces the upregulation of nuclear factor kappa-light chain enhancer of activated B-cell (NF-kB) gene, via myeloid differentiation primary response 88 (MyD88) dependent pathway, leading to the release of the first phase of pro-inflammatory mediators (PIMs) such as TNF- $\alpha$, IL-1 $\alpha$, IL-1 $\beta$, IL-6, IL-12, IL-18 and IL-23 and interferon regulatory transcription factor 3 (IRF3) gene, via MyD88 independent pathway, leading to the release of the second phase of PIMs such as IFN- $\alpha$, IFN- $\beta$, IFN- $\gamma$, IFN- $\omega$, IFN- $\varepsilon$, IFN- $\kappa$ and IFN- $\zeta$. The released PIMs such

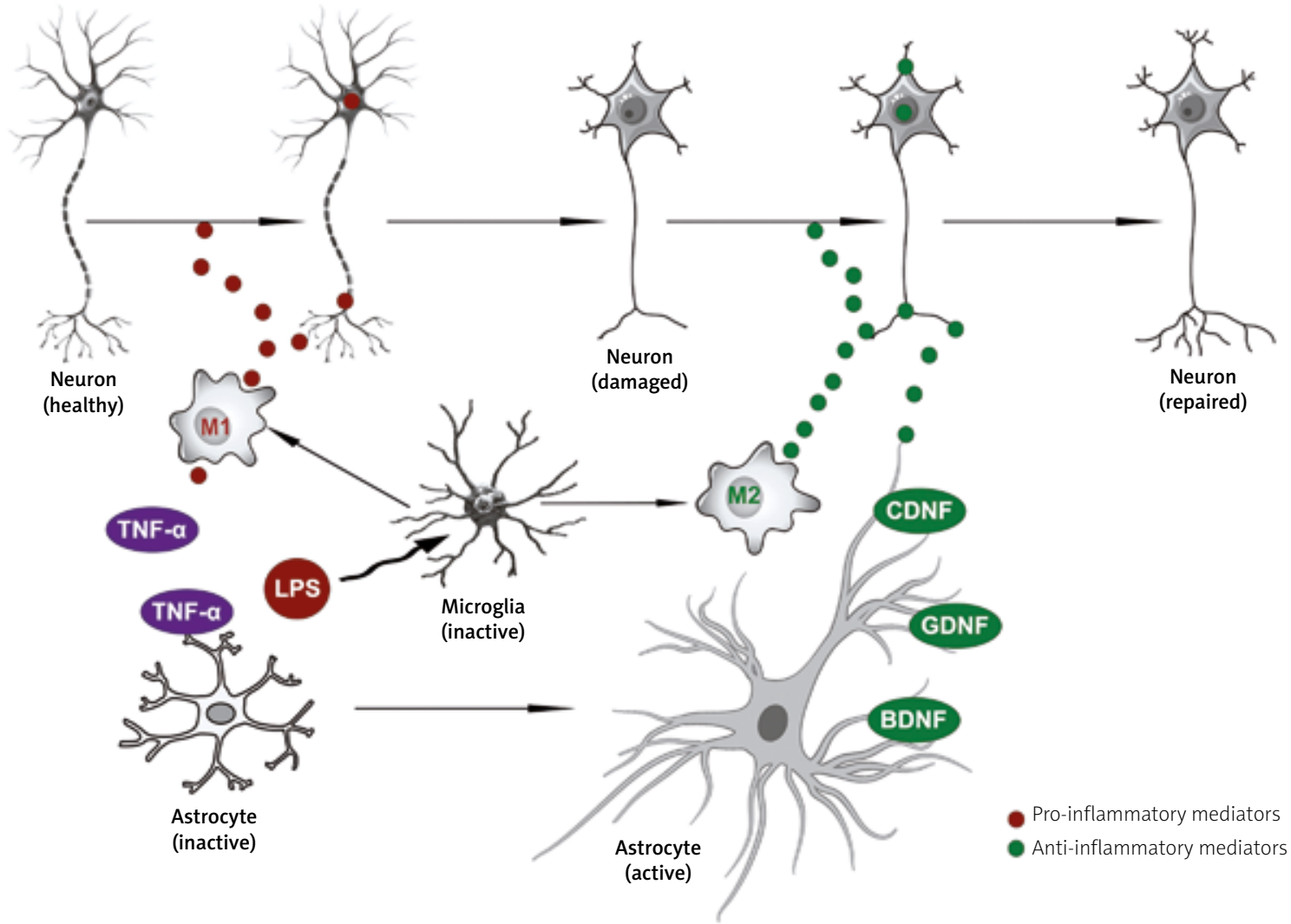

Fig. 1. Switch between neuroprotective neurotoxic microglia in an lipopolysaccharide (LPS)-related model. LPS induces the activation and conversion of inactive microglia to active M1 neurotoxic microglia, which in turn releases pro-inflammatory cytokines that are taken up by a healthy neuron in a retrograde manner and thus causes neuronal damage. The production and release of TNF- $\alpha$, also a pro-inflammatory cytokine released by microglia, induces the activation of astrocytes. Thus, activated astrocytes release large amounts of anti-inflammatory cytokines and certain neurotrophic factors, such as BDNF, CDNF and GDNF, which in turn induce the conversion of inactive microglial cells to active $M 2$ neuroprotective microglial cells. Thus, activated microglial cells also release neurotrophic factors and anti-inflammatory cytokines that are taken up by damaged or degenerated neurons and leads to the recovery of the neuron from damage induced by LPS. 

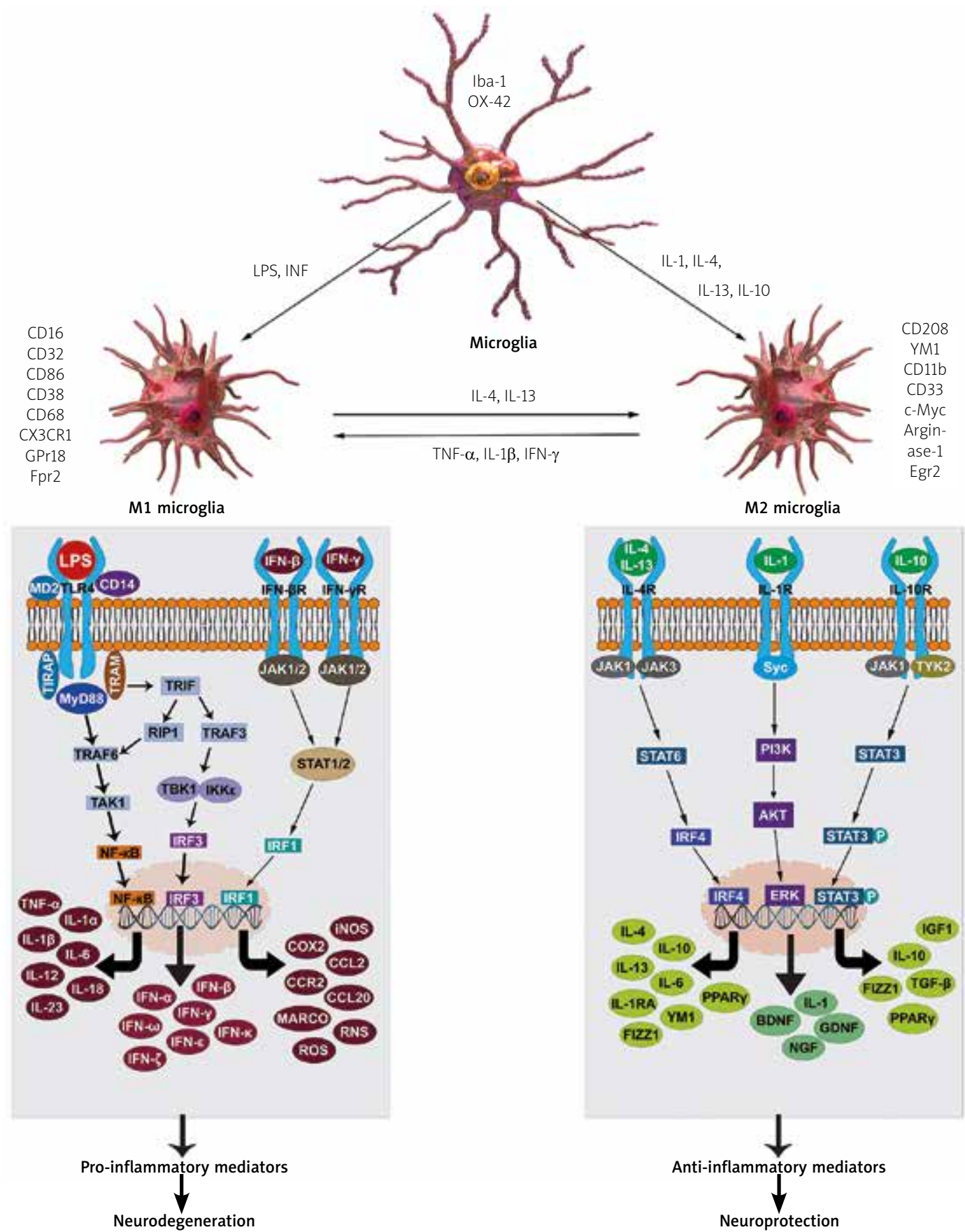

AKT - activator of protein kinase B, BDNF - brain derived neurotrophic factor, CCL- $c$ - c chemokine ligand, CCR-c - C chemokine receptor, CD - cluster of differentiation, COX - cyclooxygenase, CX3CR1 - C-X3-C motif chemokine receptor-1, EGR - early growth response, ERK - extracellular receptor kinase, GDNF - glial cell-line derived neurotrophic factor, GPR - G-protein coupled receptor, Iba - ionized calcium-binding adaptor protein, IFN - interferon, IGF - insulin-like growth factor, IKK - I kappa B kinase, IL - interleukin, iNOS - inducible nitric oxide synthase, IRF - interferon regulatory transcription factor, JAK - Janus kinase, LPS - lipopolysaccharide, MARCO - macrophage receptor with collagenous structure, MyD - myeloid differentiation factor, NGF - nerve growth factor, NF - nuclear factor, OX-42 - an antibody designed to detect CD11b, PPAR - peroxisome proliferator-activated receptor, RIP - receptor interacting protein, RNS - reactive nitrogen species, ROS - reactive oxygen species, STAT - signal transducer and activator of transcription, TAK - TGF- $\beta$ activated kinase- 1 , TBK - Tank binding kinase-1, TGF - transforming growth factor, TIR - toll interleukin-1 receptor, TIRAP - TIR domain containing adaptive protein, TLR - toll-like receptor, TNF - tumour necrosis factor, TRAF - TNF receptor associated factor, TRIF - TIR domain containing adaptor-inducing interferon $\beta$, YM-1 - neutrophil granule protein.

Fig. 2. Schematic view of lipopolysaccharide (LPS)-related cell signalling in M1 and M2 microglia subtypes. Presence of LPS or interferon (IFN) induces the activation of microglia (M1 type). Binding of LPS to toll-like receptors (TLR) and IFN- $\beta$ and IFN- $\gamma$ to their corresponding receptors (IFN- $\beta$ R and IFN- $\gamma$ R) leads to the induction of NF- $\kappa B$, IRF3 and IRF1 genes through corresponding pathways. These genes in turn lead to the production and release of huge amounts of pro-inflammatory mediators, which eventually results in the neurodegeneration. Similarly, the presence of interleukins (IL)-1, IL-4, IL-10 and IL-13 induces the activation of microglia (M2 type). Binding of IL-4, IL-13 to IL-4R, and IL-1 and IL-10 to their corresponding receptors (IL-1R and IL-10R) leads to the induction of IRF4, ERK and STAT3 genes through their corresponding pathways. These genes in turn lead to the production and release of huge amounts of anti-inflammatory mediators, which eventually results in the neuroprotection. The presence of IL-4 and IL-13 in huge amounts also switches the M1 microglia to $M 2$ microglia directing towards neuroprotection. Whereas, the presence of TNF- $\alpha$, IL- $1 \beta$ and IFN- $\gamma$ in huge amounts switches $M 2$ microglia to $M 1$ microglia and directs towards the neurodegeneration. All the markers for microglia, M1 microglia and M2 microglia are mentioned next to the image in the figure. 
as IFN- $\beta$ and IFN- $\gamma$ induce the upregulation of IRF- 1 via Janus kinase (JAK) - signal transducer and activator of transcription (STAT) pathway, leading to the release of the third/final phase of PIMs such as COX-2, chemokine ligand 2 (CCL2), c-c chemokine receptor 2 (CCR2), CCL2O, MARCO, reactive oxygen species (ROS), reactive nitrogen species (RNS) and iNOS (inducible nitric oxide synthase) $[17,33,35,63,94,95]$.

For the M2 microglia phenotype (Fig. 2), the binding of IL-4 and IL-13 to their receptors leads to the upregulation of interferon regulatory factor 4 (IRF4) gene and releases the first phase of anti-inflammatory cytokines, mediated by JAK1/3-STAT6 pathway. A second route is related to IL-1; the binding of IL-1 to its receptor leads to the induction of extracellular signal-regulated kinase (ERK) gene and releases several neurotrophic factors, mediated by protein kinase B (AKT). Similarly, the binding of IL-10 to its receptor leads to the expression of STAT3 gene and releases the third phase of anti-inflammatory mediators, mediated by JAK1/tyrosine kinase 2 (TYK2)STAT3 pathway $[21,32,51]$.

To date, several immune modulators that help in switching the microglial phenotype from $M 1$ to $M 2$ have been reported. Almost all these modulators, whether natural or other, decrease the markers and/ or pro-inflammatory mediator production and/or release and thus direct towards the M2 phenotype for protection and/or restoration purposes. Most commonly used and reported natural modulators are triterpene and stilbenes (Lupeol, Fagarsterol, Resveratrol, Malibatol A, Geraniin, Compound A (Cpd A), Paeoniflorin-6'-O-benzene sulfonate, Aloe-emoidin) $[15,53,70,71,75,100,111]$, flavonoids (Quercetin, Curcumin, Naringenin, Apigenin, Chrysin, Procyanidin, Epigallocatechin gallate, Apocynin, Paeonol) [20,27, 28,45,47,49,60,82,86,98,99,106]; terpenes (Forskolin or Coleonol, Triptolide, Terpinen-4-ol) [18,41,57,66] and alkaloid - Berberine $[23,77]$. To date, other synthetic immune modulators have been evaluated e.g. mono unsaturated fatty acid (Cis-palmitoleate) [14], Fluoroquinolone (Besifloxacin) [58], dietary supplement (Niacin) [107], Catecholamine (Dobutamine) [52], Cilostazol and $\beta$-ionone [75] in LPS-stimulated mouse alveolar macrophages. Both natural and synthetic immune modulators are evaluated in pre-clinical approaches.

\section{Conclusions}

Lipopolysaccharide-based inflammation produces neurotoxic (M1) and neuroprotective (M2) micro- glia. The M1 phenotype promotes cellular stress in the early stages of the disease, while $M 2$ microglia repair or block the brain damage progression. Some natural and synthetic modulators that could favour the $M 1 / M 2$ conversion have been reported, but robust evidence regarding cell signalling must be completed. Furthermore, the morphological changes and cytokines releasing profiles help to describe the microglial dynamics, but the proper identification of the $M 1: M 2$ ratio would represent the real challenge and position of disease, thus would better promote health of the individual.

\section{Disclosure}

The authors report no conflict of interest.

\section{References}

1. Akira S, Takeda K. Toll-like receptor signalling. Nat Rev Immunol 2004; 4: 499-511.

2. Ambrosi G, Kustrimovic N, Siani F, Rasini E, Cerri S, Ghezzi C, Dicorato G, Caputo S, Marino F, Cosentino M, Blandini F. Complex changes in the innate and adaptive immunity accompany progressive degeneration of the nigrostriatal pathway induced by intrastriatal injection of 6-hydroxydopamine in the rat. Neurotox Res 2017; 32: 71-81.

3. Augusto-Oliveira M, Arrifano GP, Lopes-Araujo A, Santos-Sacramento L, Takeda PY, Anthony DC, Malva JO, Crespo-Lopez ME. What do microglia really do in healthy adult brain? Cells 2019; 8: 1293.

4. Bai L, Zhang X, Li X, Liu N, Lou F, Ma H, Luo X, Ren Y. Somatostatin prevents lipopolysaccharide-induced neurodegeneration in the rat substantia nigra by inhibiting the activation of microglia. Mol Med Rep 2015; 12: 1002-1008.

5. Banks WA, Robinson SM. Minimal penetration of lipopolysaccharide across the murine blood-brain barrier. Brain Behav Immun 2010; 24: 102-109.

6. Batista CRA, Gomes GF, Candelario-Jalil E, Fiebich BL, de Oliveira ACP. Lipopolysaccharide-induced neuroinflammation as a bridge to understand neurodegeneration. Int J Mol Sci 2019; 20: 2293.

7. Blandini F. Neural and immune mechanisms in the pathogenesis of Parkinson's disease. J Neuroimmune Pharmacol 2013; 8: 189-201.

8. Bok E, Chung YC, Kim KS, Baik HH, Shin WH, Jin BK. Modulation of $M 1 / M 2$ polarization by capsaicin contributes to the survival of dopaminergic neurons in the lipopolysaccharide-lesioned substantia nigra in vivo. Exp Mol Med 2018; 50: 76.

9. Bossu P, Cutuli D, Palladino I, Caporali P, Angelucci F, Laricchiuta D, Gelfo F, De Bartolo P, Caltagirone C, Petrosini L. A single intraperitoneal injection of endotoxin in rats induces long-lasting modifications in behavior and brain protein levels of TNF-alpha and IL-18. J Neuroinflammation 2012; 9: 101.

10. Bozadas P, Nadella R, Sanchez-Garcia A, Rembao Bojorquez D, Rovirosa Hernandez M, Hernandez Baltazar D. Lipopolysac- 
charide-based endotoxemia produce toxicity in peripheral organs and microglia migration in a dose-dependent manner in rat substantia nigra. Folia Neuropathol 2019; 57: 258-266.

11. Campos FL, Carvalho MM, Cristovao AC, Je G, Baltazar G, Salgado AJ, Kim YS, Sousa N. Rodent models of Parkinson's disease: beyond the motor symptomatology. Front Behav Neurosci 2013; 7: 175.

12. Castano A, Herrera AJ, Cano J, Machado A. The degenerative effect of a single intranigral injection of LPS on the dopaminergic system is prevented by dexamethasone, and not mimicked by rh-TNF-alpha, IL-1beta and IFN-gamma. J Neurochem 2002; 81: 150-157.

13. Catorce MN, Gevorkian G. LPS-induced murine neuroinflammation model: main features and suitability for pre-clinical assessment of nutraceuticals. Curr Neuropharmacol 2016; 14 : 155-164

14. Chan KL, Pillon NJ, Sivaloganathan DM, Costford SR, Liu Z, Theret M, Chazaud B, Klip A. Palmitoleate reverses high fat-induced proinflammatory macrophage polarization via AMP-activated protein kinase (AMPK). J Biol Chem 2015; 290: 16979. 16988.

15. Chang $Y$, Jia X, Wei F, Wang C, Sun X, Xu S, Yang X, Zhao Y, Chen J, Wu H, Zhang L, Wei W. CP-25, a novel compound, protects against autoimmune arthritis by modulating immune mediators of inflammation and bone damage. Sci Rep 2016; 6: 26239.

16. Chen L, Liu P, Feng X, Ma C. Salidroside suppressing LPS-induced myocardial injury by inhibiting ROS-mediated PI3K/ Akt/mTOR pathway in vitro and in vivo. J Cell Mol Med 2017; 21: 3178-3189.

17. Chen Z, Jalabi W, Shpargel KB, Farabaugh KT, Dutta R, Yin X, Kidd GJ, Bergmann CC, Stohlman SA, Trapp BD. Lipopolysaccharide-induced microglial activation and neuroprotection against experimental brain injury is independent of hematogenous TLR4. J Neurosci 2012; 32: 11706-11715.

18. Chiadak JD, Arsenijevic T, Verstrepen K, Gregoire F, Bolaky N, Delforge V, Flamand V, Perret J, Delporte C. Forskolin inhibits lipopolysaccharide-induced modulation of MCP-1 and GPR120 in 3T3-L1 adipocytes through an inhibition of NFkappaB. Mediators Inflamm 2016; 2016: 1431789.

19. Choi DY, Liu M, Hunter RL, Cass WA, Pandya JD, Sullivan PG, Shin EJ, Kim HC, Gash DM, Bing G. Striatal neuroinflammation promotes Parkinsonism in rats. PLoS One 2009; 4: e5482.

20. Chou TC. Anti-inflammatory and analgesic effects of paeonol in carrageenan-evoked thermal hyperalgesia. $\mathrm{Br} J$ Pharmacol 2003; 139: 1146-1152

21. Cochet F, Peri F. The role of carbohydrates in the lipopolysaccharide (LPS)/Toll-like receptor 4 (TLR4) signalling. Int J Mol Sci 2017; 18: 2318

22. Cryan JF, O'Riordan KJ, Cowan CSM, Sandhu KV, Bastiaanssen TFS, Boehme M, Codagnone MG, Cussotto S, Fulling C, Golubeva AV, Guzzetta KE, Jaggar M, Long-Smith CM, Lyte JM, Martin JA, Molinero-Perez A, Moloney G, Morelli E, Morillas E, O'Connor R, Cruz-Pereira JS, Peterson VL, Rea K, Ritz NL, Sherwin E, Spichak S, Teichman EM, van de Wouw M, VenturaSilva AP, Wallace-Fitzsimons SE, Hyland N, Clarke G, Dinan TG.
The microbiota-gut-brain axis. Physiol Rev 2019; 99: 18772013.

23. Cui G, Qin X, Zhang Y, Gong Z, Ge B, Zang YQ. Berberine differentially modulates the activities of ERK, p38 MAPK, and JNK to suppress Th17 and Th1 T cell differentiation in type 1 diabetic mice. J Biol Chem 2009; 284: 28420-28429.

24. Cunningham C. Microglia and neurodegeneration: the role of systemic inflammation. Glia 2013; 61: 71-90.

25. DiSabato DJ, Quan N, Godbout JP. Neuroinflammation: the devil is in the details. J Neurochem 2016; 139 Suppl 2: 136-153.

26. Durafourt BA, Moore CS, Zammit DA, Johnson TA, Zaguia F, Guiot MC, Bar-Or A, Antel JP. Comparison of polarization properties of human adult microglia and blood-derived macrophages. Glia 2012; 60: 717-727.

27. Feng X, Qin H, Shi Q, Zhang Y, Zhou F, Wu H, Ding S, Niu Z, Lu Y, Shen $P$. Chrysin attenuates inflammation by regulating M1/M2 status via activating PPARgamma. Biochem Pharmacol 2014; 89: 503-514.

28. Feng X, Weng D, Zhou F, Owen YD, Qin H, Zhao J, WenYu, Huang Y, Chen J, Fu H, Yang N, Chen D, Li J, Tan R, Shen P. Activation of PPARgamma by a natural flavonoid modulator, apigenin ameliorates obesity-related inflammation via regulation of macrophage polarization. EBioMedicine 2016; 9: 61-76.

29. Firmino M, Weis SN, Souza JMF, Gomes BRB, Mol AR, Mortari MR, Souza GEP, Coca GC, Williams TCR, Fontes W, Ricart CAO, de Sousa MV, Veiga-Souza FH. Label-free quantitative proteomics of rat hypothalamus under fever induced by LPS and PGE2. J Proteomics 2018; 187: 182-199.

30. Flores-Martinez YM, Fernandez-Parrilla MA, Ayala-Davila J, Reyes-Corona D, Blanco-Alvarez VM, Soto-Rojas LO, LunaHerrera C, Gonzalez-Barrios JA, Leon-Chavez BA, GutierrezCastillo ME, Martinez-Davila IA, Martinez-Fong D. Acute neuroinflammatory response in the substantia nigra pars compacta of rats after a local injection of lipopolysaccharide. J Immunol Res 2018; 2018: 1838921.

31. Fu HQ, Yang T, Xiao W, Fan L, Wu Y, Terrando N, Wang TL. Prolonged neuroinflammation after lipopolysaccharide exposure in aged rats. PLoS One 2014; 9: e106331.

32. Funami K, Matsumoto M, Oshiumi H, Inagaki F, Seya T. Functional interfaces between TICAM-2/TRAM and TICAM-1/TRIF in TLR4 signaling. Biochem Soc Trans 2017; 45: 929-935.

33. Gao T, Chen Z, Chen H, Yuan H, Wang Y, Peng X, Wei C, Yang J, $\mathrm{Xu}$ C. Inhibition of HMGB1 mediates neuroprotection of traumatic brain injury by modulating the microglia/macrophage polarization. Biochem Biophys Res Commun 2018; 497: 430436.

34. Gelders G, Baekelandt V, Van der Perren A. Linking neuroinflammation and neurodegeneration in Parkinson's disease. J Immunol Res 2018; 2018: 4784268.

35. Ghosh M, Xu Y, Pearse DD. Cyclic AMP is a key regulator of M1 to $M 2 a$ phenotypic conversion of microglia in the presence of Th2 cytokines. J Neuroinflammation 2016; 13: 9.

36. Ginhoux F, Greter M, Leboeuf M, Nandi S, See P, Gokhan S, Mehler MF, Conway SJ, Ng LG, Stanley ER, Samokhvalov IM, Merad M. Fate mapping analysis reveals that adult microglia derive from primitive macrophages. Science 2010; 330: 841-845. 
37. Godbout JP, Chen J, Abraham J, Richwine AF, Berg BM, Kelley $\mathrm{KW}$, Johnson RW. Exaggerated neuroinflammation and sickness behavior in aged mice following activation of the peripheral innate immune system. FASEB J 2005; 19: 1329-1331.

38. Gottschall PE, Komaki G, and Arimura A. Increased circulating interleukin-1 and interleukin-6 after intracerebroventricular injection of lipopolysaccharide. Neuroendocrinology 1992; 56: 935-938.

39. Granholm AC, Zaman V, Godbee J, Smith M, Ramadan R, Umphlet C, Randall P, Bhat NR, Rohrer B, Middaugh LD, Boger HA Prenatal LPS increases inflammation in the substantia nigra of Gdnf heterozygous mice. Brain Pathol 2011; 21: 330-348.

40. Hakuno F, Takahashi SI. IGF1 receptor signaling pathways. J Mol Endocrinol 2018; 61: T69-T86.

41. Hart PH, Brand C, Carson CF, Riley TV, Prager RH, Finlay-Jones JJ. Terpinen-4-ol, the main component of the essential oil of Melaleuca alternifolia (tea tree oil), suppresses inflammatory mediator production by activated human monocytes. Inflamm Res 2000; 49: 619-626.

42. Hernandez-Baltazar D, Zavala-Flores LM, Villanueva-Olivo A The 6-hydroxydopamine model and parkinsonian pathophys iology: Novel findings in an older model. Neurologia 2017; 32 533-539.

43. Hopp SC, Royer SE, D’Angelo HM, Kaercher RM, Fisher DA, Wenk GL. Differential neuroprotective and anti-inflammatory effects of L-type voltage dependent calcium channel and ryanodine receptor antagonists in the substantia nigra and locus coeruleus. J Neuroimmune Pharmacol 2015; 10: 35-44.

44. Huang B, Liu J, Meng T, Li Y, He D, Ran X, Chen G, Guo W, Kan X, Fu S, Wang W, Liu D. Polydatin prevents lipopolysaccharide (LPS)-induced Parkinson's disease via regulation of the AKT/ GSK3beta-Nrf2/NF-kappaB signaling axis. Front Immunol 2018; 9: 2527

45. Huang L, Zhang B, Yang Y, Gong X, Chen Z, Wang Z, Zhang P, Zhang Q. Synthesis and anti-inflammatory activity of paeonol analogues in the murine model of complete Freund's adjuvant induced arthritis. Bioorg Med Chem Lett 2016; 26 : 5218-5221.

46. lizumi T, Takahashi S, Mashima K, Minami K, Izawa Y, Abe T, Hishiki T, Suematsu M, Kajimura M, Suzuki N. A possible role of microglia-derived nitric oxide by lipopolysaccharide in activation of astroglial pentose-phosphate pathway via the Keap1/ Nrf2 system. J Neuroinflammation 2016; 13: 99.

47. Impellizzeri D, Esposito E, Mazzon E, Paterniti I, Di Paola R, Bramanti P, Cuzzocrea S. Effect of apocynin, a NADPH oxidase inhibitor, on acute lung inflammation. Biochem Pharmacol 2011; 81: 636-648.

48. Joseph B, Venero JL. A brief overview of multitalented microglia. Methods Mol Biol 2013; 1041: 3-8.

49. Kim SY, Moon KA, Jo HY, Jeong S, Seon SH, Jung E, Cho YS, Chun E, Lee KY. Anti-inflammatory effects of apocynin, an inhibitor of NADPH oxidase, in airway inflammation. Immunol Cell Biol 2012; 90: 441-448.

50. Kolac UK, Ustuner MC, Tekin N, Ustuner D, Colak E, Entok E. The anti-inflammatory and antioxidant effects of salvia officinalis on lipopolysaccharide-induced inflammation in rats. J Med Food 2017; 20: 1193-1200.
51. Kuzmich NN, Sivak KV, Chubarev VN, Porozov YB, SavateevaLyubimova TN, Peri F. TLR4 signaling pathway modulators as potential therapeutics in inflammation and sepsis. Vaccines (Basel) 2017; 5: 34.

52. Li CY, Tsai CS, Hsu PC, Wu CT, Wong CS, Ho ST. Dobutamine modulates lipopolysaccharide-induced macrophage inflammatory protein-1alpha and interleukin-8 production in human monocytes. Anesth Analg 2003; 97: 210-215.

53. Liberman AC, Antunica-Noguerol M, Ferraz-de-Paula V, Palermo-Neto J, Castro CN, Druker J, Holsboer F, Perone MJ, Gerlo S, De Bosscher K, Haegeman G, Arzt E. Compound A, a dissociated glucocorticoid receptor modulator, inhibits T-bet (Th1) and induces GATA-3 (Th2) activity in immune cells. PLoS One 2012; 7: e35155.

54. Lim KH, Staudt LM. Toll-like receptor signaling. Cold Spring Harb Perspect Biol 2013; 5: a011247.

55. Liu M, Bing G. Lipopolysaccharide animal models for Parkinson's disease. Parkinsons Dis 2011; 2011: 327089.

56. Lopes PC. LPS and neuroinflammation: a matter of timing. Inflammopharmacology 2016; 24: 291-293.

57. Lu Y, Liu Y, Fukuda K, Nakamura Y, Kumagai N, Nishida T. Inhibition by triptolide of chemokine, proinflammatory cytokine, and adhesion molecule expression induced by lipopolysaccharide in corneal fibroblasts. Invest Ophthalmol Vis Sci 2006; 47: 3796-3800.

58. Mah FS, Sanfilippo CM. Besifloxacin: efficacy and safety in treatment and prevention of ocular bacterial infections. Ophthalmol Ther 2016; 5: 1-20.

59. Mantovani A, Biswas SK, Galdiero MR, Sica A, Locati M. Macrophage plasticity and polarization in tissue repair and remodelling. J Pathol 2013; 229: 176-185.

60. Martinez-Micaelo N, Gonzalez-Abuin N, Ardevol A, Pinent M, Blay MT. Procyanidins and inflammation: molecular targets and health implications. Biofactors 2012; 38: 257-265.

61. Meireles M, Marques C, Norberto S, Santos P, Fernandes I, Mateus N, Faria A, Calhau C. Anthocyanin effects on microglia M1/M2 phenotype: Consequence on neuronal fractalkine expression. Behav Brain Res 2016; 305: 223-228.

62. Mendes-Oliveira J, Lopes Campos F, Videira RA, Baltazar G. GPER activation is effective in protecting against inflammation-induced nigral dopaminergic loss and motor function impairment. Brain Behav Immun 2017; 64: 296-307.

63. Miao H, Li R, Han C, Lu X, Zhang H. Minocycline promotes posthemorrhagic neurogenesis via M2 microglia polarization via upregulation of the TrkB/BDNF pathway in rats. J Neurophysiol 2018; 120: 1307-1317.

64. Mogensen TH. Pathogen recognition and inflammatory signaling in innate immune defenses. Clin Microbiol Rev 2009; 22: 240-273.

65. Nguyen TA, Frank-Cannon T, Martinez TN, Ruhn KA, Marvin M, Casey B, Trevino I, Hong JJ, Goldberg MS, Tansey MG. Analysis of inflammation-related nigral degeneration and locomotor function in DJ-1(-/-) mice. J Neuroinflammation 2013; 10: 50.

66. Nogueira MN, Aquino SG, Rossa Junior C, Spolidorio DM. Terpinen-4-ol and alpha-terpineol (tea tree oil components) inhibit the production of IL-1beta, IL- 6 and IL-10 on human macrophages. Inflamm Res 2014; 63: 769-778. 
67. Okada K, Arai S, Itoh H, Adachi S, Hayashida M, Nakase H, Ikemoto M. CD68 on rat macrophages binds tightly to S100A8 and S100A9 and helps to regulate the cells' immune functions. J Leukoc Biol 2016; 100: 1093-1104.

68. Orihuela R, McPherson CA, Harry GJ. Microglial M1/M2 polarization and metabolic states. Br J Pharmacol 2016; 173: 649665.

69. Palsson-McDermott EM, O’Neill LA. Signal transduction by the lipopolysaccharide receptor, Toll-like receptor-4. Immunology 2004; 113: 153-162.

70. Pan J, Jin JL, Ge HM, Yin KL, Chen X, Han LJ, Chen Y, Qian L, Li XX $X u$ Y. Malibatol A regulates microglia M1/M2 polarization in experimental stroke in a PPARgamma-dependent manner. J Neuroinflammation 2015; 12: 51.

71. Park MY, Kwon HJ, Sung MK. Evaluation of aloin and aloeemodin as anti-inflammatory agents in aloe by using murine macrophages. Biosci Biotechnol Biochem 2009; 73: 828-832.

72. Proniewski B, Kij A, Sitek B, Kelley EE, Chlopicki S. Multiorgan development of oxidative and nitrosative stress in LPS-induced endotoxemia in C57Bl/6 mice: DHE-based in vivo approach. Oxid Med Cell Longev 2019; 2019: 7838406.

73. Rabenstein M, Vay SU, Blaschke S, Walter HL, Ladwig A, Fink GR, Rueger MA, Schroeter M. Crosstalk between stressed brain cells: direct and indirect effects of ischemia and aglycemia on microglia. J Neuroinflammation 2020; 17: 33.

74. Rappold PM, Cui M, Chesser AS, Tibbett J, Grima JC, Duan L, Sen N, Javitch JA, Tieu K. Paraquat neurotoxicity is mediated by the dopamine transporter and organic cation transporter-3. Proc Natl Acad Sci U S A 2011; 108: 20766-20771.

75. Saqib U, Sarkar S, Suk K, Mohammad O, Baig MS, Savai R. Phytochemicals as modulators of M1-M2 macrophages in inflammation. Oncotarget 2018; 9: 17937-17950.

76. Serdar M, Kempe K, Rizazad M, Herz J, Bendix I, FelderhoffMuser U, Sabir H. Early pro-inflammatory microglia activation after inflammation-sensitized hypoxic-ischemic brain injury in neonatal rats. Front Cell Neurosci 2019; 13: 237.

77. Shang W, Liu J, Yu X, and Zhao J. Effects of berberine on serum levels of inflammatory factors and inflammatory signaling pathway in obese mice induced by high fat diet. Zhongguo Zhong Yao Za Zhi 2010; 35: 1474-1477.

78. Sharma N, Nehru B. Characterization of the lipopolysaccharide induced model of Parkinson's disease: Role of oxidative stress and neuroinflammation. Neurochem Int 2015; 87: 92-105.

79. Shi H, Wang XL, Quan HF, Yan L, Pei XY, Wang R, Peng XD. Effects of betaine on LPS-stimulated activation of microglial M1/M2 phenotypes by suppressing TLR4/NF-kappaB pathways in N9 cells. Molecules 2019; 24: 367.

80. Shpargel KB, Jalabi W, Jin Y, Dadabayev A, Penn MS, Trapp BD. Preconditioning paradigms and pathways in the brain. Cleve Clin J Med 2008; 75 Suppl 2: S77-82.

81. Singh AK, Jiang Y. How does peripheral lipopolysaccharide induce gene expression in the brain of rats? Toxicology 2004; 201: 197-207.

82. Singh NA, Mandal AK, Khan ZA. Potential neuroprotective properties of epigallocatechin-3-gallate (EGCG). Nutr J 2016; 15: 60 .
83. Sochocka M, Diniz BS, Leszek J. Inflammatory response in the CNS: friend or foe? Mol Neurobiol 2017; 54: 8071-8089.

84. Steimle A, Autenrieth IB, Frick JS. Structure and function: lipid A modifications in commensals and pathogens. Int J Med Microbiol 2016; 306: 290-301.

85. Sugita H, Kaneki M, Tokunaga E, Sugita M, Koike C, Yasuhara S, Tompkins RG, Martyn JA. Inducible nitric oxide synthase plays a role in LPS-induced hyperglycemia and insulin resistance. Am J Physiol Endocrinol Metab 2002; 282: E386-394.

86. Sun GY, Chen Z, Jasmer KJ, Chuang DY, Gu Z, Hannink M, Simonyi A. Quercetin attenuates inflammatory responses in BV-2 microglial cells: role of MAPKs on the Nrf2 pathway and induction of heme oxygenase-1. PLoS One 2015; 10: e0141509.

87. Sun J, Zhang S, Zhang X, Zhang X, Dong H, Qian Y. IL-17A is implicated in lipopolysaccharide-induced neuroinflammation and cognitive impairment in aged rats via microglial activation. J Neuroinflammation 2015; 12: 165.

88. Sun XC, Ren XF, Chen L, Gao XQ, Xie JX, Chen WF. Glucocorticoid receptor is involved in the neuroprotective effect of ginsenoside Rg1 against inflammation-induced dopaminergic neuronal degeneration in substantia nigra. J Steroid Biochem Mol Biol 2016; 155: 94-103.

89. Tanaka T, Murakami K, Bando Y, Yoshida S. Interferon regulatory factor 7 participates in the M1-like microglial polarization switch. Glia 2015; 63: 595-610.

90. Thiruchelvam M, Brockel BJ, Richfield EK, Baggs RB, CorySlechta DA. Potentiated and preferential effects of combined paraquat and maneb on nigrostriatal dopamine systems: environmental risk factors for Parkinson's disease? Brain Res 2000; 873: 225-234.

91. Vay SU, Flitsch LJ, Rabenstein M, Rogall R, Blaschke S, Kleinhaus J, Reinert N, Bach A, Fink GR, Schroeter M, Rueger MA. The plasticity of primary microglia and their multifaceted effects on endogenous neural stem cells in vitro and in vivo. J Neuroinflammation 2018; 15: 226.

92. Walker DG, Lue LF. Immune phenotypes of microglia in human neurodegenerative disease: challenges to detecting microglial polarization in human brains. Alzheimers Res Ther 2015; 7: 56.

93. Wang GQ, Li DD, Huang C, Lu DS, Zhang C, Zhou SY, Liu J, Zhang F. Icariin reduces dopaminergic neuronal loss and microgliamediated inflammation in vivo and in vitro. Front Mol Neurosci 2018; 10: 441.

94. Wang S, Wang F, Yang H, Li R, Guo H, Hu L. Diosgenin glucoside provides neuroprotection by regulating microglial M1 polarization. Int Immunopharmacol 2017; 50: 22-29.

95. Wang Y, Huang Y, Xu Y, Ruan W, Wang H, Zhang Y, Saavedra JM, Zhang L, Huang Z, Pang T. A dual AMPK/Nrf2 activator reduces brain inflammation after stroke by enhancing microglia $\mathrm{m} 2$ polarization. Antioxid Redox Signal 2018; 28: 141-163.

96. Wang YW, Zhou Q, Zhang X, Qian QQ, Xu JW, Ni PF, Qian YN. Mild endoplasmic reticulum stress ameliorates lipopolysaccharide-induced neuroinflammation and cognitive impairment via regulation of microglial polarization. J Neuroinflammation 2017; 14: 233.

97. Wu F, Luo T, Mei Y, Liu H, Dong J, Fang Y, Peng J, Guo Y. Simvastatin alters M1/M2 polarization of murine BV2 microglia via Notch signaling. J Neuroimmunol 2018; 316: 56-64. 
98. Wu LH, Lin C, Lin HY, Liu YS, Wu CY, Tsai CF, Chang PC, Yeh WL, Lu DY. Naringenin suppresses neuroinflammatory responses through inducing suppressor of cytokine signaling 3 expression. Mol Neurobiol 2016; 53: 1080-1091.

99. Yadav R, Jee B, Awasthi SK. Curcumin suppresses the production of pro-inflammatory cytokine interleukin-18 in lipopolysaccharide stimulated murine macrophage-like cells. Indian J Clin Biochem 2015; 30: 109-112.

100. Yang X, Xu S, Qian Y, Xiao Q. Resveratrol regulates microglia M1/M2 polarization via PGC-1alpha in conditions of neuroinflammatory injury. Brain Behav Immun 2017; 64: 162-172.

101. Zhang B, Wei YZ, Wang GQ, Li DD, Shi JS, Zhang F. Targeting MAPK pathways by naringenin modulates microglia M1/M2 polarization in lipopolysaccharide-stimulated cultures. Front Cell Neurosci 2018; 12: 531.

102. Zhang J, Stanton DM, Nguyen XV, Liu M, Zhang Z, Gash D, Bing G. Intrapallidal lipopolysaccharide injection increases iron and ferritin levels in glia of the rat substantia nigra and induces locomotor deficits. Neuroscience 2005; 135: 829-838.

103. Zhang L, Previn R, Lu L, Liao RF, Jin Y, Wang RK. Crocin, a natural product attenuates lipopolysaccharide-induced anxiety and depressive-like behaviors through suppressing NF-kB and NLRP3 signaling pathway. Brain Res Bull 2018; 142: 352-359.

104. Zhang L, Zhang J, Jiang X, Yang L, Zhang Q Wang B, Cui L, Wang $X$. Hydroxytyrosol inhibits LPS-induced neuroinflammatory responses via suppression of TLR-4-mediated NF-kappaB P65 activation and ERK signaling pathway. Neuroscience 2020; 426: 189-200.

105. Zheng Y, He R, Wang P, Shi Y, Zhao L, Liang J. Exosomes from LPS-stimulated macrophages induce neuroprotection and functional improvement after ischemic stroke by modulating microglial polarization. Biomater Sci 2019; 7: 2037-2049.

106. Zhong Y, Chiou YS, Pan MH, Shahidi F. Anti-inflammatory activity of lipophilic epigallocatechin gallate (EGCG) derivatives in LPS-stimulated murine macrophages. Food Chem 2012; 134: 742-748.

107. Zhou E, Li Y, Yao M, Wei Z, Fu Y, Yang Z. Niacin attenuates the production of pro-inflammatory cytokines in LPS-induced mouse alveolar macrophages by HCA2 dependent mechanisms. Int Immunopharmacol 2014; 23: 121-126.

108. Zhou T, Huang Z, Sun X, Zhu X, Zhou L, Li M, Cheng B, Liu X, He C. Microglia polarization with M1/M2 phenotype changes in rd1 mouse model of retinal degeneration. Front Neuroanat 2017; $11: 77$

109. Zhou Y, Wang G, Li D, Wang Y, Wu Q, Shi J, Zhang F. Dual modulation on glial cells by tetrahydroxystilbene glucoside protects against dopamine neuronal loss. J Neuroinflammation 2018; 15: 161.

110. Zhu Y, Carvey PM, Ling Z. Altered glutathione homeostasis in animals prenatally exposed to lipopolysaccharide. Neurochem Int 2007; 50: 671-680.

111. Zhu Y, Li X, Chen J, Chen T, Shi Z, Lei M, Zhang Y, Bai P, Li Y, Fei $X$. The pentacyclic triterpene Lupeol switches M1 macrophages to $\mathrm{M} 2$ and ameliorates experimental inflammatory bowel disease. Int Immunopharmacol 2016; 30: 74-84. 